\title{
Manufacturing Execution Systems: Examples of Performance Indicator and Operational Robustness Tools
}

\author{
Yannick Gendre*, Gérard Waridel, Myrtille Guyon, Jean-François Demuth, Hervé Guelpa, \\ and Thierry Humbert
}

\begin{abstract}
Manufacturing Execution Systems (MES) are computerized systems used to measure production performance in terms of productivity, yield, and quality. In the first part, performance indicator and overall equipment effectiveness (OEE), process robustness tools and statistical process control are described. The second part details some tools to help process robustness and control by operators by preventing deviations from target control charts. MES was developed by Syngenta together with CIMO for automation.
\end{abstract}

Keywords: Manufacturing Execution System · OEE · Process robustness · Production performance . Statistical process control

\section{Introduction}

Automation in the chemical process industry has allowed more productivity and generated lots of data. Automatic processing of these data is needed for continuous improvement of productivity and quality. Some methods and concepts for productivity improvement and robustness tools will be described and illustrated in this article, starting with performance indicators and cycle time followed by examples of statistical process control on quality. Finally real-time monitoring and examples of process deviation will be detailed.

\section{Overall Equipment Effectiveness and Performance Indicators}

Overall Equipment Effectiveness (OEE) is a common performance indicator used in industry, which is based on three categories: Availability, Performance and Quality (Eqn. (1)). This article illustrates how Management Execution System (MES) can be used to monitor OEE for batch production and continuous production.

OEE $=$ Performance $\times$ Availability $\times$ Quality [\%]
OEE is monitored only at the production line bottleneck. This first section will focus on Performance and Availability. Quality measurement and statistics are described in another section.

\subsection{Batch Production}

According to International Society of Automation (ISA) Standard $88,{ }^{[1]}$ batch production is defined as a succession of operations. OEE is measured at the unit with the highest cycle time of the production line which is the bottleneck.

For batch production, Performance is measured for each batch and is equal to the ratio between target batch cycle time (OEE $100 \%$ ) versus measured batch cycle time. This ratio cannot be higher than $100 \%$. The time difference between target and current operation time is recorded as a delay. As the target OEE calculation method is not well defined in the literature, ${ }^{[2]}$ the follow- ing method is used. Target cycle time (or minimum achievable cycle time) is the sum of the minimum time of each operation. According to our observation target cycle time is lower by $1-5 \%$ than minimum measured cycle time.

A batch cycle time representation example is given in Fig. 1 where the batch cycle time is divided into operation times. Each operation can be then displayed individually (Fig. 2), helping the identification of production loss root causes. The performance of each operation in the batch is measured and consolidated to give the performance of the entire batch.

The background green zones in Figs 1 and 2 represent the minimum operation time OEE $100 \%$. When measurements are displayed in the orange zone, it demonstrates that a minor deviation was recorded. When they reach the red zone, it shows that a major deviation occurred.

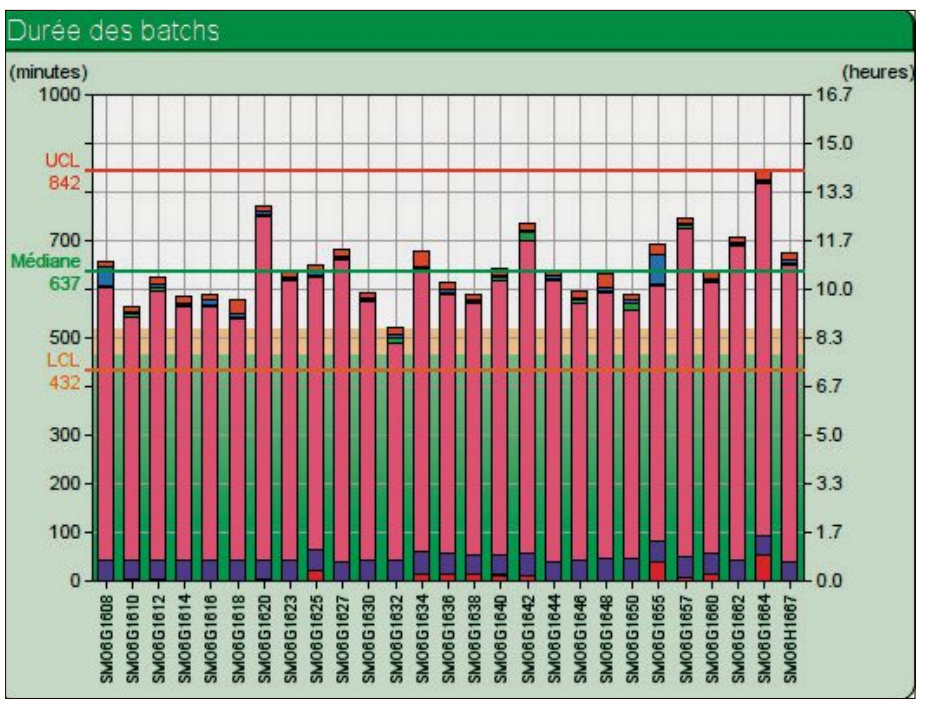

Fig. 1. Batch cycle time histogram visualisation. 


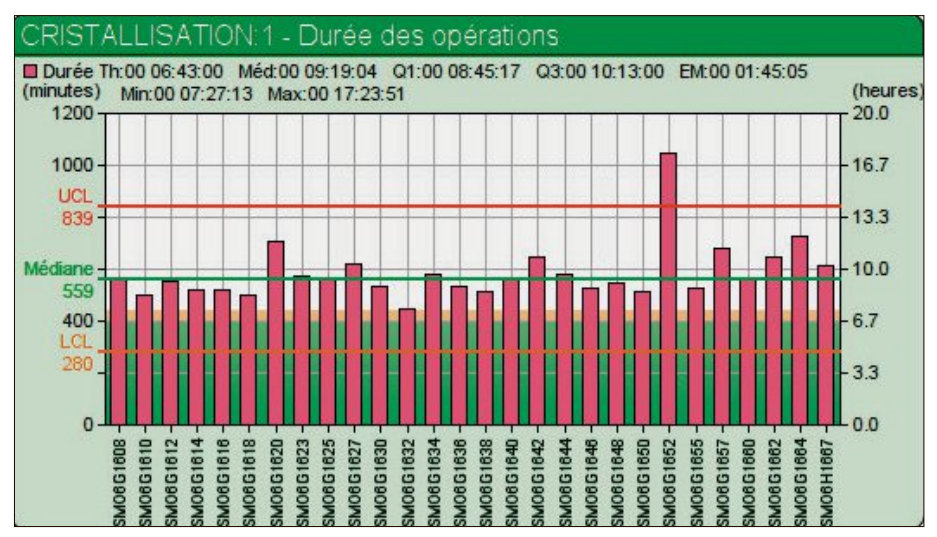

Fig. 2. Operation cycle time histogram.

Major OEE loss records should be analysed by the operational team to ensure productivity robustness. Minor deviations are generally not analysed.

The cycle time evolution can be followed using a moving average. The variability can be monitored with basic statistical tools such as mean value, standard deviation, minimum and maximum values...

\subsection{Continuous Production}

For continuous production, productivity is measured using product flow rate at the input or output of the unit. Performance is the ratio of measured versus target flow rate.

Availability is defined as the ratio of operating time/planned production time. Loss of availability is detected when the flow is equal to zero. This happens when no production is planned on the equipment and this record should not be treated. Fig. 3 represents the schematic view of productivity loss for continuous process.

In Fig. 4 continuous flow rate versus time is displayed. OEE target flow is $5200 \mathrm{~kg} / \mathrm{h}$. Each flow rate reduction or stop generates an OEE loss, which is then summarized in a table.

\subsection{OEE Productivity Loss Analysis}

Once OEE is measured either on a batch or a continuous production, a production record loss table is generated. The cause of each production loss must be assigned to a specific cause by the operational team.

An analysis of the main causes of production loss can then be performed on different time frames (weekly, monthly) which allows continuous improvement of productivity. Fig. 5 illustrates an example of a weekly OEE productivity loss report split into different categories.

\section{Quality: Statistical Process Control on Defects}

Quality can be monitored using MES following direct data or variability of quality parameters.

The main quality indicator is Right
First Time (RFT), which represents the amount of product aligned with the specifications. RFT evolution versus time can be monitored. To improve the product compliance rate, the cause of defects should be analysed: the number of noncompliant criteria or near misses (within specification but between than $80 \%$ and $99 \%$ of minimum or maximum specification limits) are counted and visualised in a pie plot to aid defect cause identification (Fig. 6).

After RFT tracking and defect causes identification, the process variability is measured. The variability is monitored using average and moving average of the finished product (or by-product content), standard deviation and 6-sigma indica-

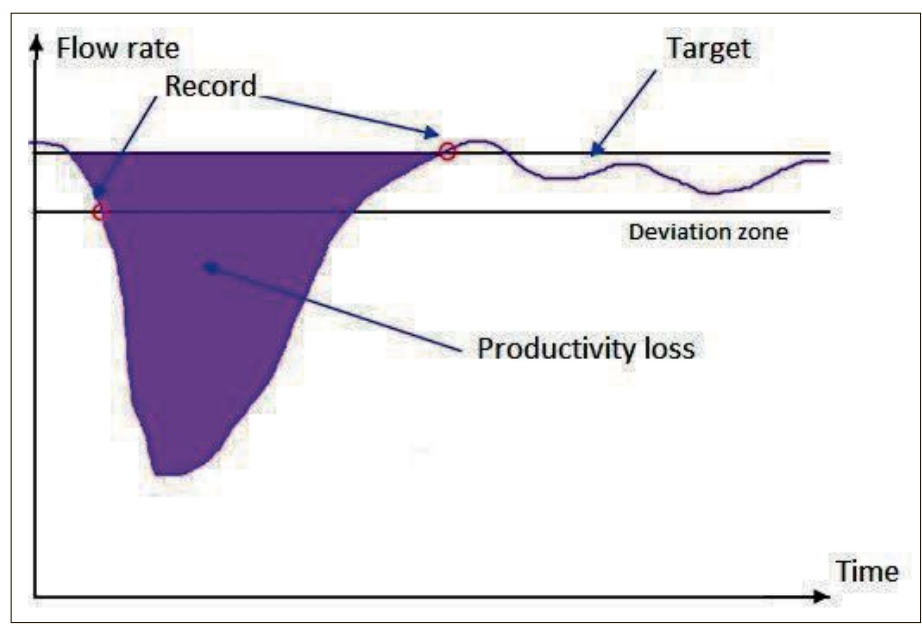

Fig. 3. Schematic view of loss of productivity flow for continuous process.

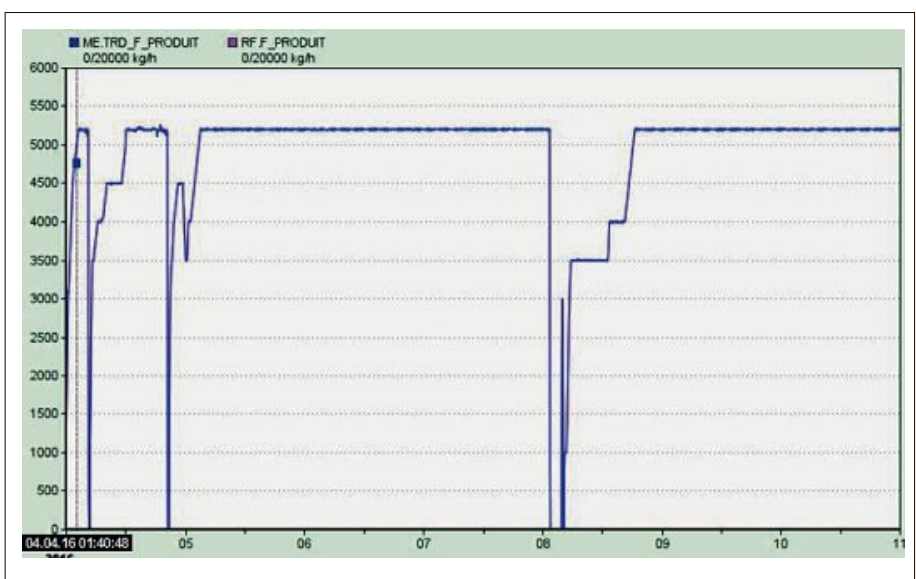

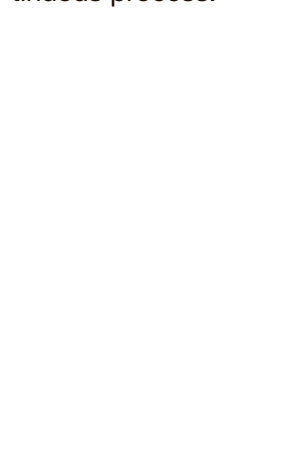

Fig. 4. Continuous productivity loss. tors (Process Performance $\mathrm{Pp}=$ Upper Specification Limit - Lower Specification Limit/6 Standard deviation and Process Capability Cp). Fig. 7 illustrates a well-controlled by-product with a Process Capability Upper (Cpu) higher than 1.67 (typical over quality value according to 6-sigma Pillet M, 2011). ${ }^{[3]}$

\section{Mass Balance and Yield Measurement}

Mass balance and yield process performance can be monitored using MES. Each chemical consumed or produced is declared in the MES database which allows the calculation of the ratio between chemical production and raw material consumption. Moreover the assay can be extracted from Laboratory Information Management System (LIMS), giving such values for each consumed and produced chemical.

Fig. 8 and Fig. 9 represent the evolution of produced and consumed material quantities, respectively, versus target on a monthly basis or batch per batch.

Using the above data, the ratio of consumed raw material versus produced chemicals can be calculated and allows efficiency tracking and deviation control. 


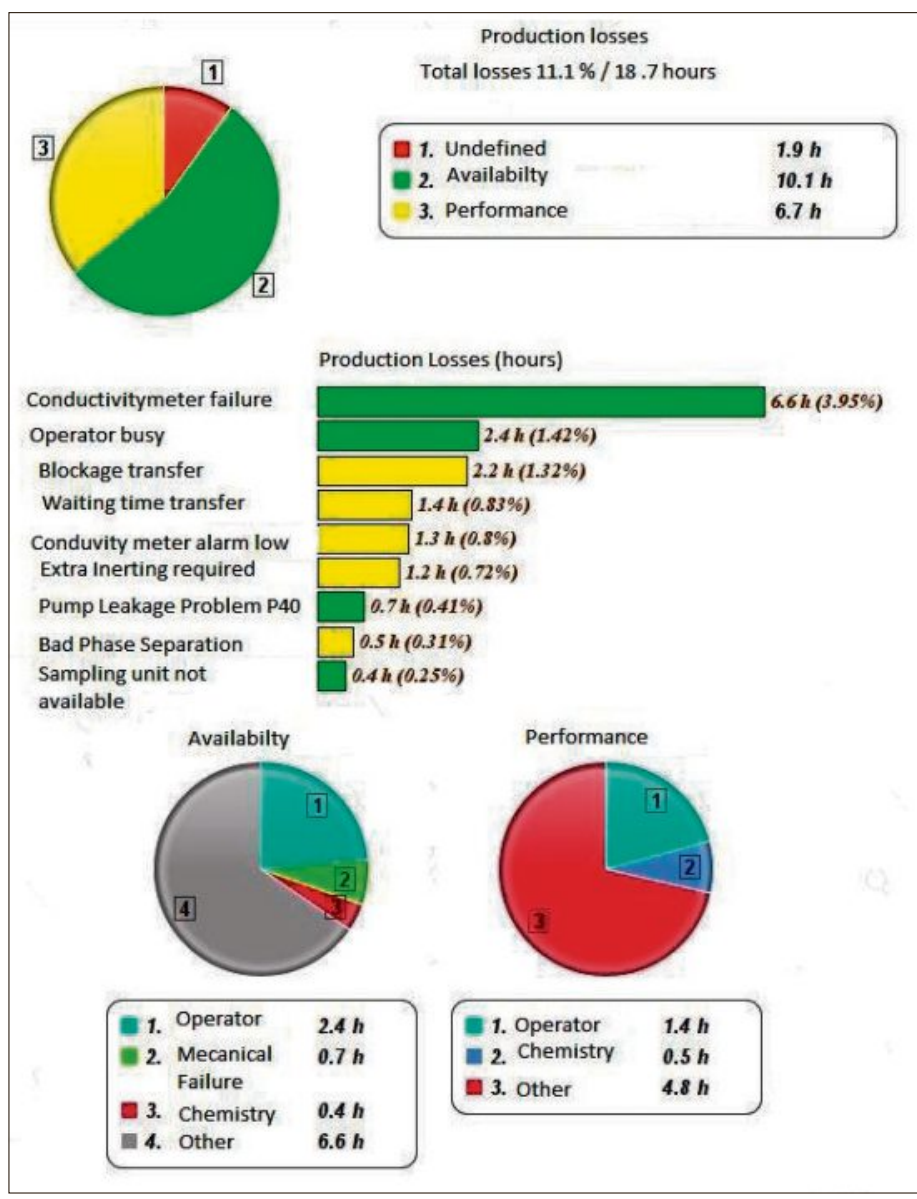

Fig. 5. OEE Loss Pareto analysis.

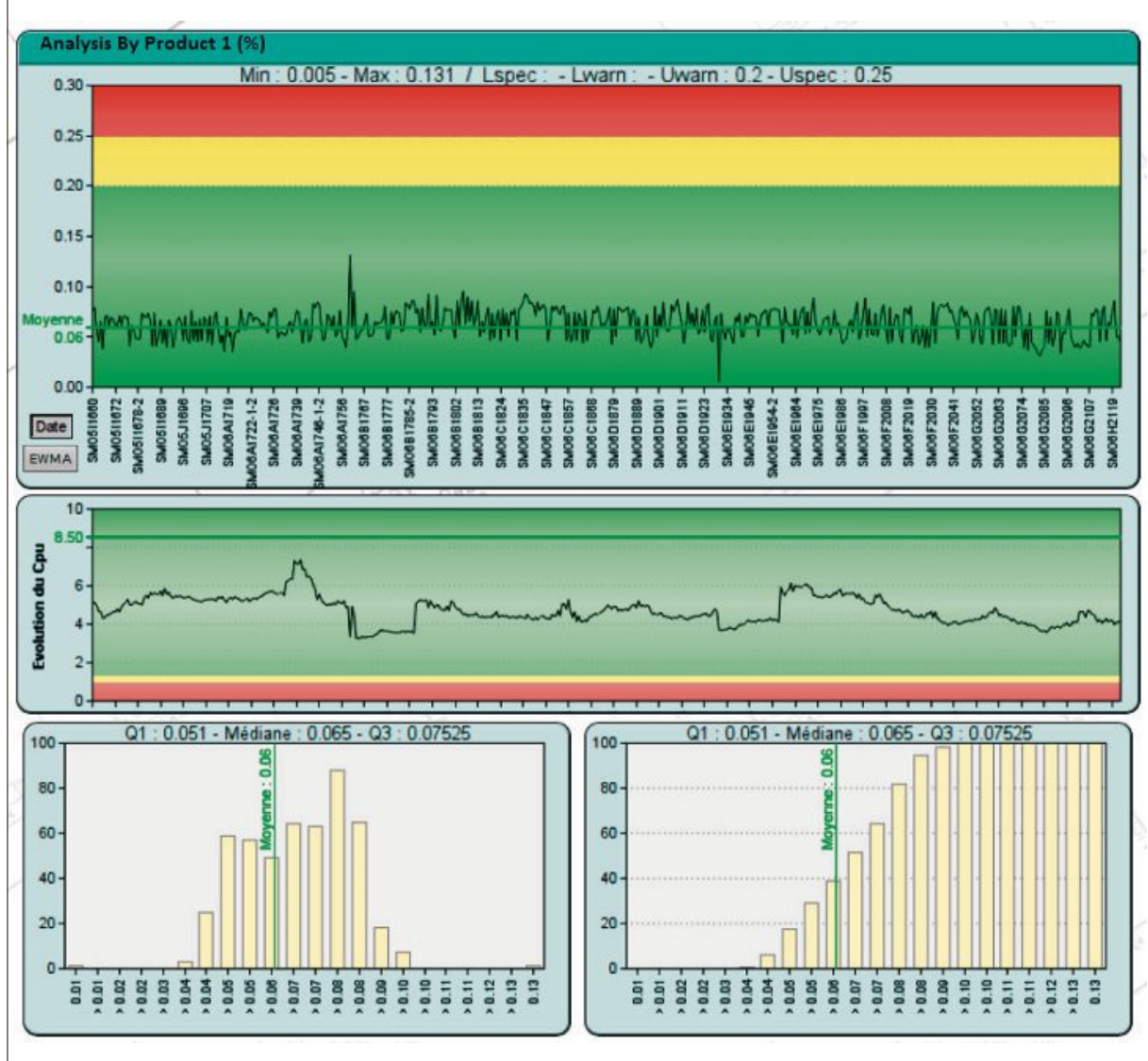

Fig. 7. (Top) Evolution of by-product versus batch number with $0.25 \%$ upper limit and average; (Centre) evolution of capability versus batch number with limits and average; (Bottom left) population distribution by occurrence of by-product value; (Bottom right) cumulative population histogram in \%.

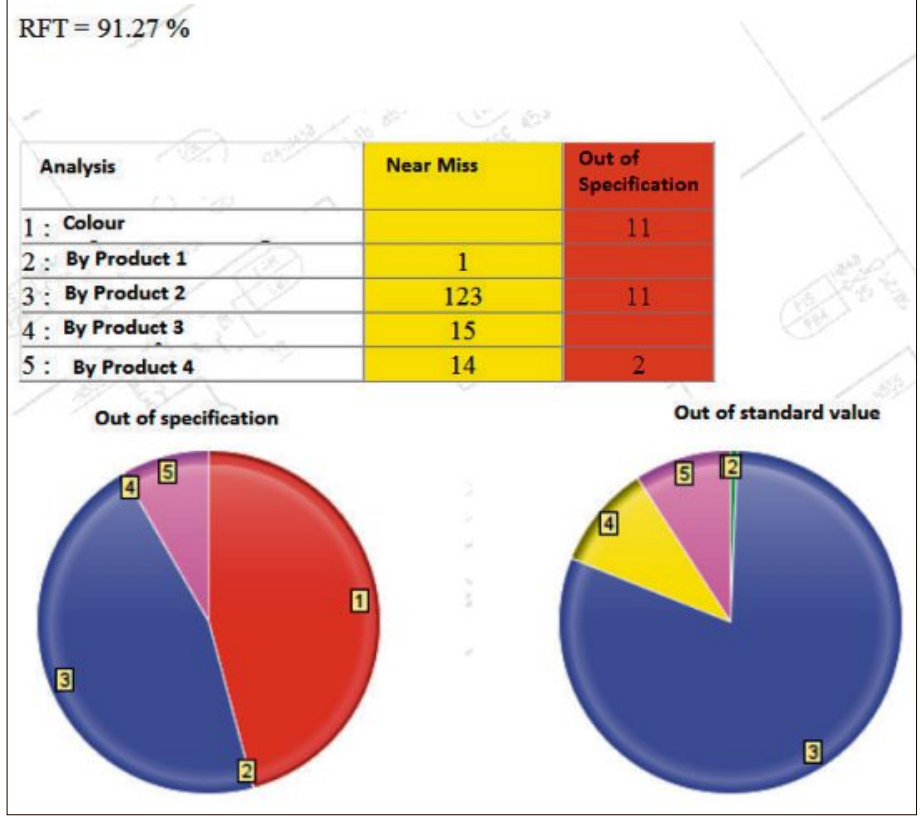

Fig. 6. Quality analysis on noncompliance criteria occurrence.

\section{Execution Supervision and Process Robustness}

Production dashboards are used to monitor production performance live. Such tools help to control the process as it allows early deviation detection to keep them as low as possible.

\subsection{Continuous Production Robustness}

Typical dashboards used to monitor plant performance are speedometers with high/low limits and histograms. Parameters which are followed are direct measures: flow rate, temperature, $\mathrm{pH}$... and indirect measures such as mass yield (ratio of flow in/out) versus rate for yield calculation or quality.

Fig. 10 and Fig. 11 illustrate the flow rate of the continuous evaporator with the yield of evaporation using those speedometers.

For a parameter that has to be constant versus time, horizontal working zones are commonly used. For example, temperature can be monitored during raw material dosing in continuous or batch production. The standard conditions are displayed in green. Small deviations are displayed in yellow and main deviations are in red. Fig. 12 represents constant $\mathrm{pH}$ monitoring with working zones.

In the case of deviation from the optimal working zone, action needs to be taken, which are then detailed on screen to fix the problem. Fig. 13 is an example of such process deviation from standard operating conditions and the key actions to be taken. 


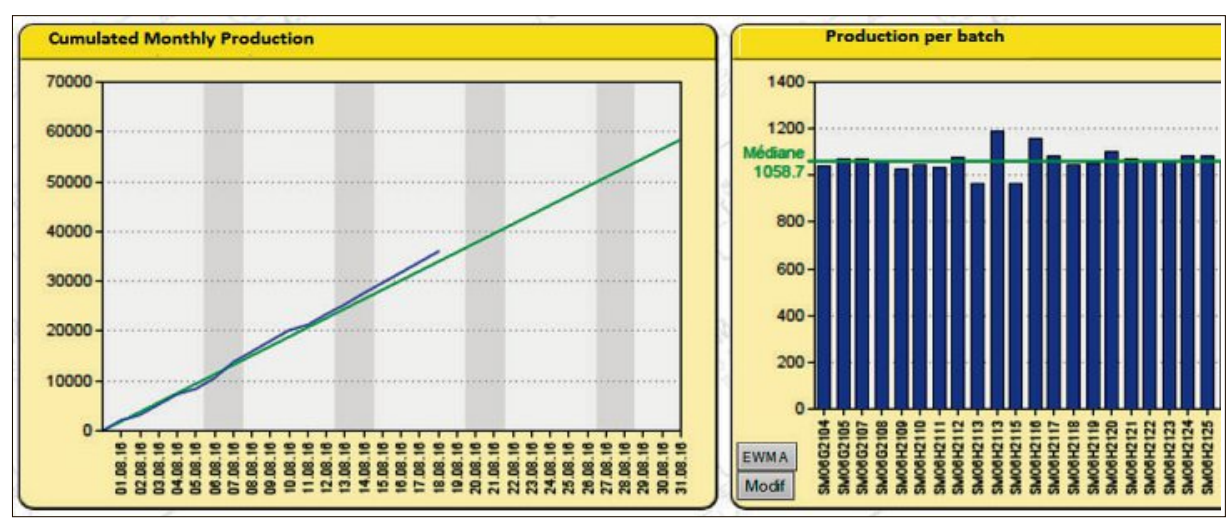

Fig. 8. (Left) Production evolution in blue compared to planning in green versus time on monthly base; (Right) Batch production quantity representation versus batch number.

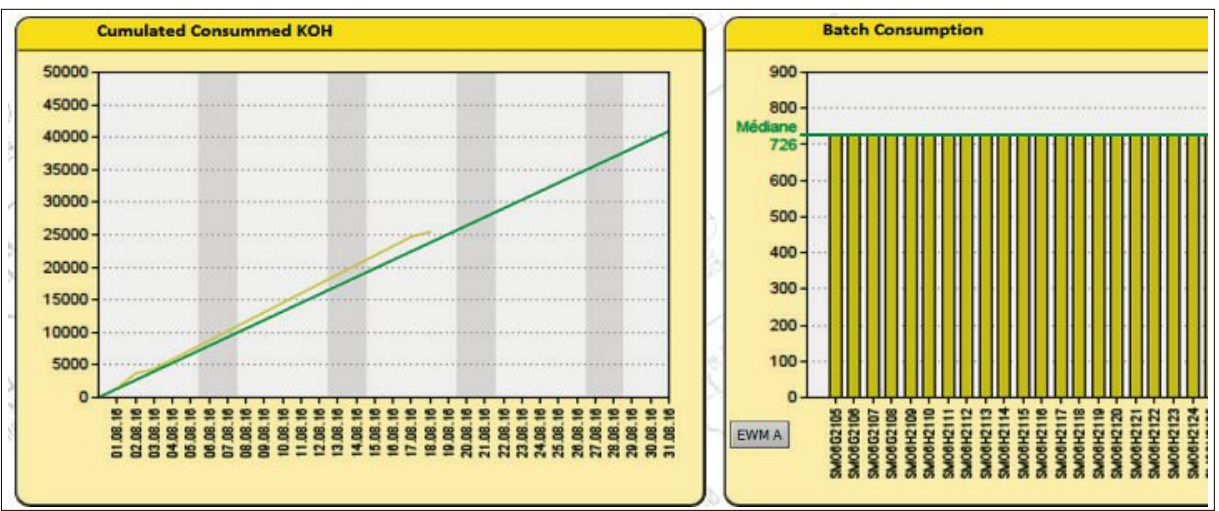

Fig. 9. (Left) Raw material consumption evolution in yellow compared to planning in green versus time on monthly base; (Right) Batch consumption quantity representation versus batch number.

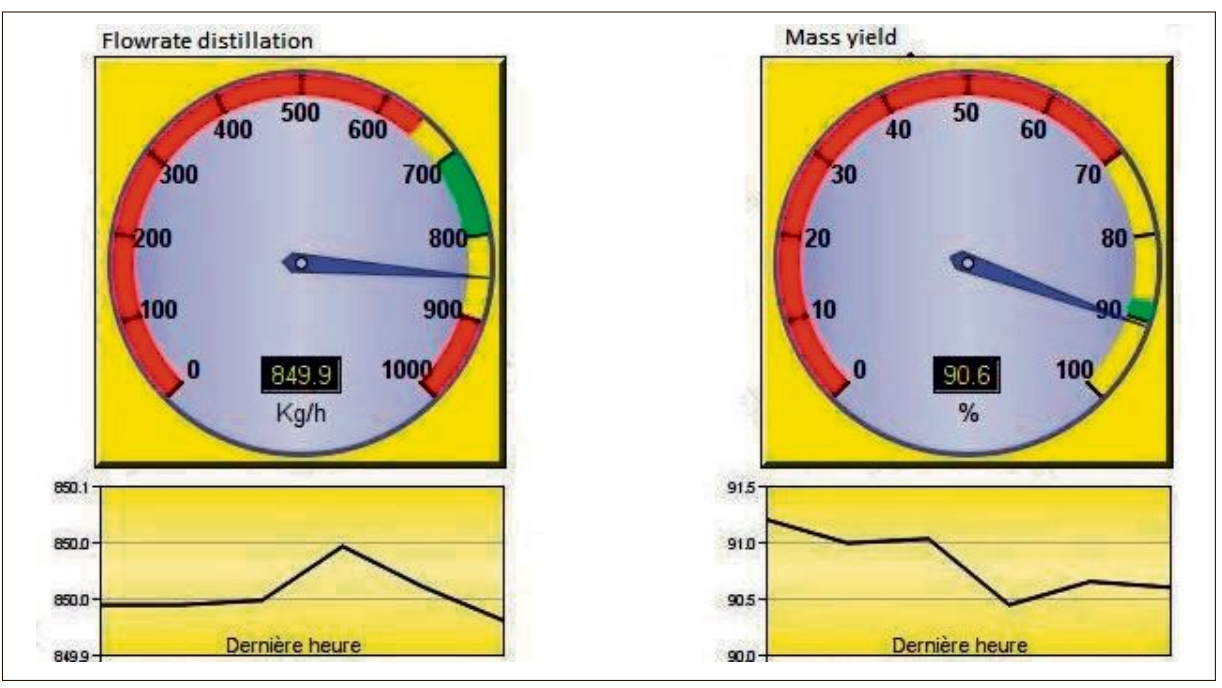

Fig. 10. Flow and yield dashboard on continuous evaporation unit.

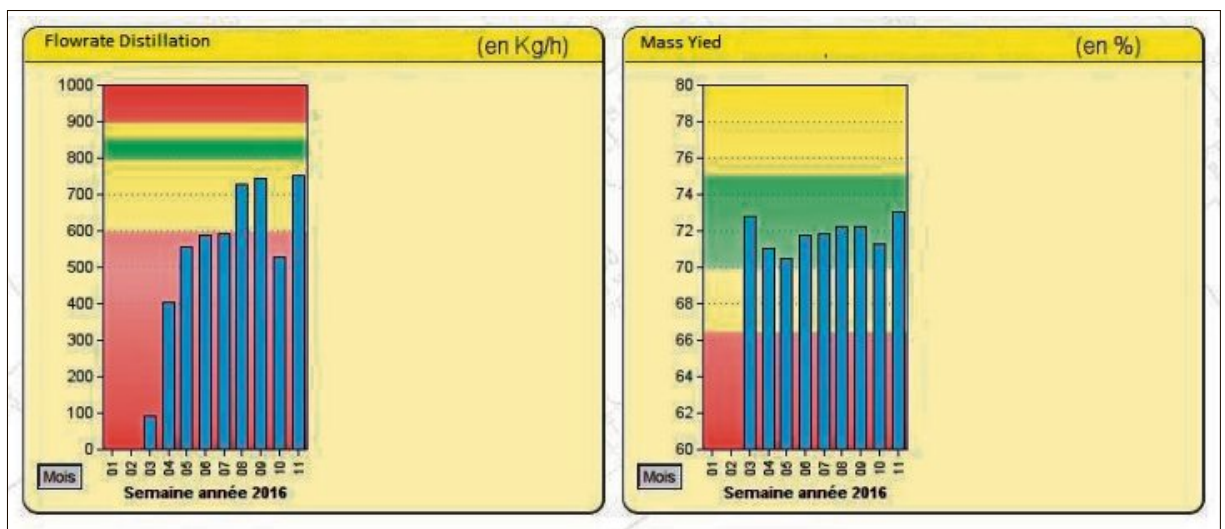

Fig. 11. Weekly dashboard on continuous evaporation unit.

\subsection{Batch Production Robustness}

For batch production, a reference called the 'golden batch' must be replicated to ensure productivity and quality. Typical parameters which could be followed are temperature, pressure, cumulated dosed, distilled chemical...

Fig. 14 illustrates a crystallisation cooling temperature profile compared to a reference with a band of deviation. In case of deviation, the temperature curve leaves the green and yellow band to enter the red deviation zone.

Alarms are recorded as process deviations. The time spent outside the standard working zone is recorded as well. The deviation causes should be analysed further to improve reproducibility. Such a graph helps the operators to follow a dynamic profile, which can prevent a minor or a major deviation.

In Fig. 15, a cooling profile deviation at a low temperature of $-5{ }^{\circ} \mathrm{C}$ occurred, caused by brine network overconsumption. Brine power consumption was exceeding the chiller power and the reactor could not be cooled at the expected rate.

The main process control parameter was the amount of each chemical dosed per batch to ensure reproducibility. In Fig. 16 , histogram representation of the amount dosed per batch with deviation helped operators to establish the limits. Further statistics can then be performed to monitor the variability. This type of histogram is displayed during each batch dosing step.

\section{Conclusion and Outlook}

In this article, some examples of MES were presented to facilitate deviation monitoring and to prevent such deviations from becoming significant. These tools can help productivity and economic performance measurement and to control the process better. It was observed that using such indicators engages the operational team in the continuous improvement process by empowering them in the decision-making process. It was observed that using all of these tools led in two to three years to more than ten percent productivity increase and fewer process deviations.

Received: June 2, 2016 
[1] ANSI/ISA-88.01-2010 Batch Control Part 1: Models and terminology Ansi/Isa.

[2] NF E60-182, Afnor, 2002.

[3] M. Pillet, 'Six Sigma: Comment l'appliquer', Eyrolles, Paris, 2011.

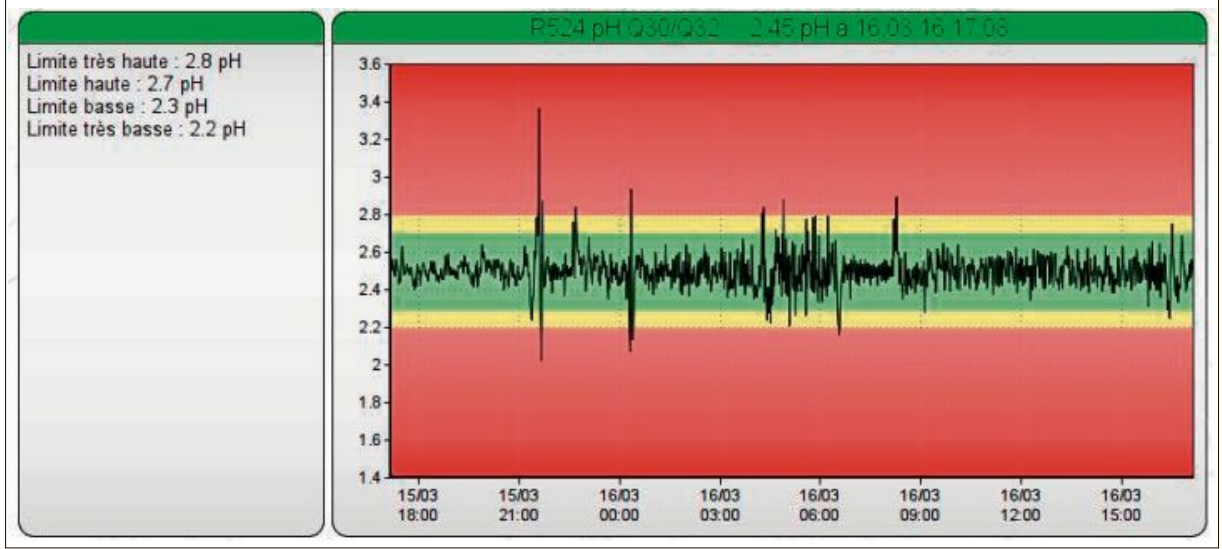

Fig. 12. Constant $\mathrm{pH}$ monitoring with control limit.

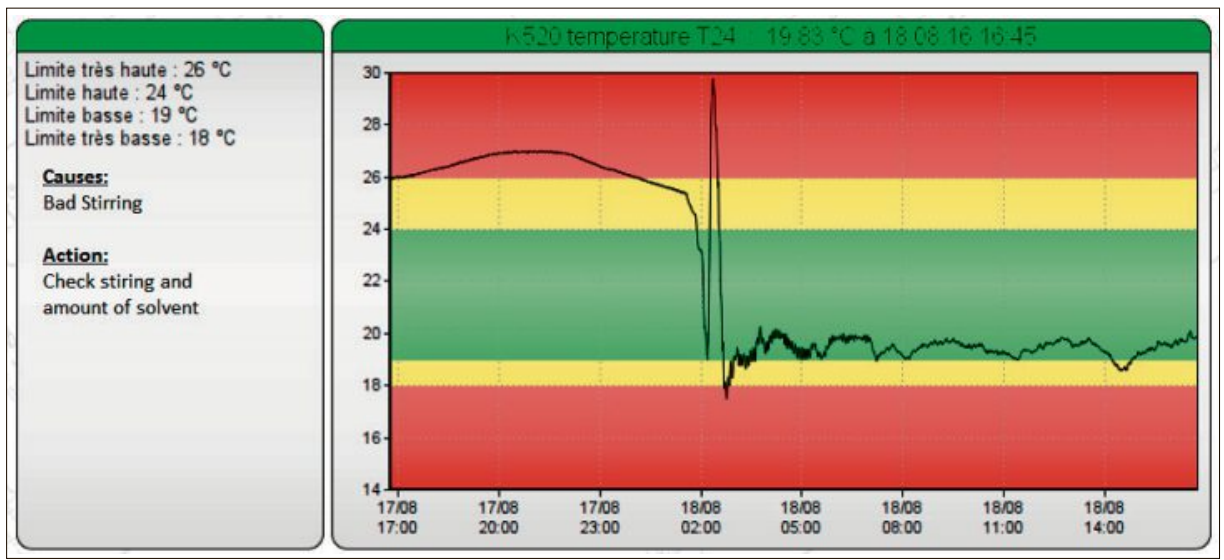

Fig. 13. Constant temperature monitoring with detected deviation and actions to be followed.
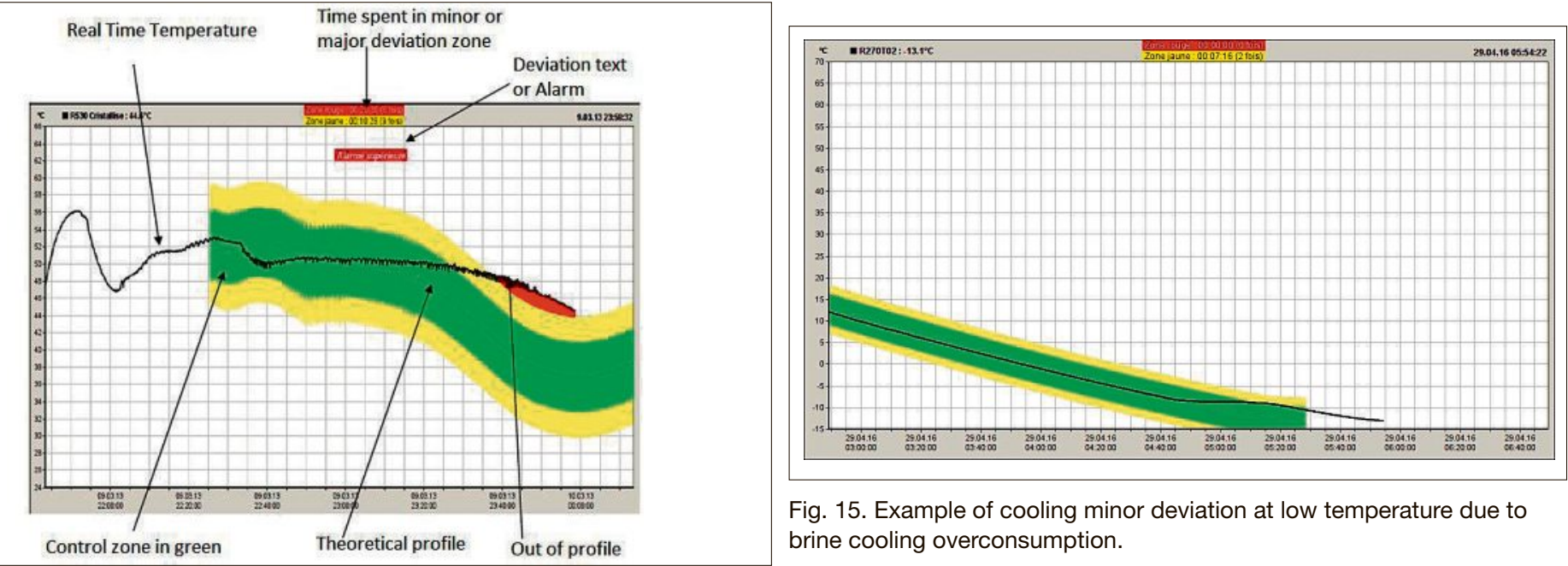

Fig. 15. Example of cooling minor deviation at low temperature due to brine cooling overconsumption.
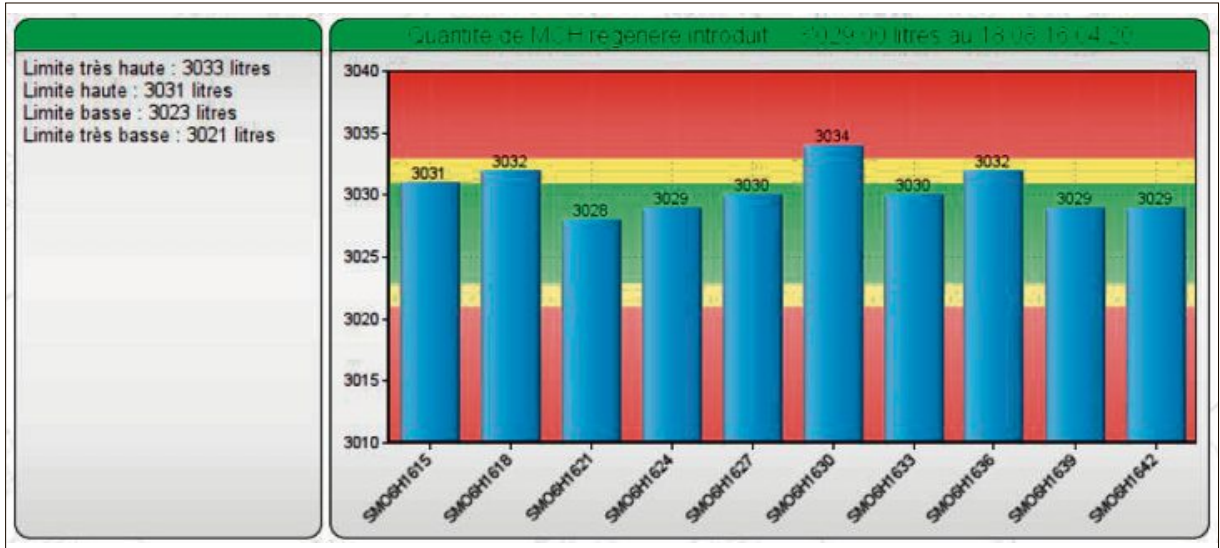

Fig. 16. Histogram representation of chemical dosed with deviation zone.
Fig. 14. Golden Batch temperature profile with major deviation. 